\title{
Electronic band structure and Fermi surface of ferromagnetic Tb: Experiment and theory
}

\author{
K. M. Döbrich, ${ }^{1, *}$ G. Bihlmayer, ${ }^{2}$ K. Starke, ${ }^{1, \dagger}$ J. E. Prieto, ${ }^{1,+}$ K. Rossnagel,${ }^{3,}$ H. Koh, ${ }^{3}$ E. Rotenberg, ${ }^{3}$ S. Blügel, ${ }^{2}$ and \\ G. Kaindl ${ }^{1}$ \\ ${ }^{1}$ Institut für Experimentalphysik, Freie Universität Berlin, Arnimallee 14, 14195 Berlin-Dahlem, Germany \\ ${ }^{2}$ Institut für Festkörperforschung, Forschungszentrum Jülich, 52425 Jülich, Germany \\ ${ }^{3}$ Advanced Light Source, Lawrence Berkeley National Laboratory, Berkeley, California 94720, USA
}

(Received 20 December 2006; revised manuscript received 15 May 2007; published 30 July 2007)

\begin{abstract}
We have investigated the bulk valence-band structure of $\mathrm{Tb}$ metal in the ferromagnetic phase by angleresolved photoelectron spectroscopy and full-potential-linearized-augmented-plane-wave calculations. The experiments were performed at undulator beamline 7.0.1 of the Advanced Light Source using a three-axis rotatable low-temperature goniometer and a display-type photoelectron spectrometer that give access to a large region of momentum space. The results of our calculations, which make use of recent progress in the theoretical description of the magnetic properties of $4 f$ metals, are in remarkably good agreement with experiment. This can be best seen from a comparison of the electronic structure in high-symmetry directions, at critical points, on Fermi contours, and at band crossings with the Fermi level. To our knowledge, the present work represents the most detailed combined experimental and theoretical study of the electronic structure of a magnetic lanthanide metal to date.
\end{abstract}

DOI: 10.1103/PhysRevB.76.035123

PACS number(s): 79.60.-i, 71.18. + y, 71.20.Eh

\section{INTRODUCTION}

The electronic band structure of a solid, and in particular the crossings of electronic states in $\mathbf{k}$ space with the Fermi energy (the Fermi surface), determine its low-energy excitation properties at finite temperatures that are relevant, e.g., for electrical conductivity, specific heat, and magnetic susceptibility. ${ }^{1-3}$ For lanthanide metals, the shape of the Fermi surface (FS) is of particular interest, since the magnetic $4 f$ moments are highly localized, with negligible overlap of the $4 f$ orbitals on nearest-neighbor (NN) atoms. In these materials, long-range magnetic order can only be mediated in an indirect way via polarization of the conduction electrons, i.e., by Ruderman-Kittel-Kasuya-Yosida (RKKY) interaction. Since this magnetic interaction is highly sensitive to the specific properties of the FS, minor differences of the electronic structure and of the FS in the various lanthanide metals lead to a great variety of magnetic behavior, such as the development of paramagnetic, ferromagnetic, helical antiferromagnetic (AFM), and conical ferrimagnetic phases, depending on temperature and on the particular lanthanide metal. ${ }^{4}$

Many of the experimental and theoretical studies on the relationship between magnetic order and electronic structure of lanthanides focus on Gd metal, ${ }^{5-13}$ which represents a prototypical local-moment Heisenberg ferromagnet. Some studies also deal with $\mathrm{Tb}$, Dy, and Ho metals. ${ }^{14-22}$ There are also a few reports on the electronic structure of the hexagonal close-packed (hcp) Y metal, ${ }^{23,24}$ which are of interest in the present context, due to similarities in the band structure of $Y$ metal with the paramagnetic bands of the hcp heavy lanthanide metals, including Tb (see also Refs. 25-27 that report on FSs of $\mathrm{Y}, \mathrm{Gd}$, and $\mathrm{Y}_{x} \mathrm{Gd}_{1-x}$ alloys). Most previous photoemission (PE) studies on heavy lanthanide metals have analyzed the band structure along the $\Gamma A$ high-symmetry direction of the hexagonal Brillouin zone (BZ), i.e., in normal emission from (0001)-oriented single crystals (see Fig. 1).
Lanthanide-metal films with this orientation can be grown with very good single-crystalline structure and high chemical purity.

As far as the status of the theoretical description of the electronic structure of lanthanide metals is concerned, it is fair to say that most theoretical calculations date back to the late 1960 s and early 1970s, when non-spin-polarized calculations were performed to study the Fermi surfaces of the paramagnetic phases. Since then, most work has focused on Gd due to its half-filled $4 f$ shell. Certain aspects, such as magnetic anisotropy, however, have been studied also for other $4 f$ elements, while studies of band structures and FSs are rare (some bands are presented in Ref. 21, but a complete picture is missing). Since the magnetic coupling in lanthanide metals depends strongly on the energies of the electronic bands and on their crossings with the Fermi energy, it is crucial to know how well the band structure of a given lanthanide metal is described by theory. Recently developed theoretical methods to improve the description of the

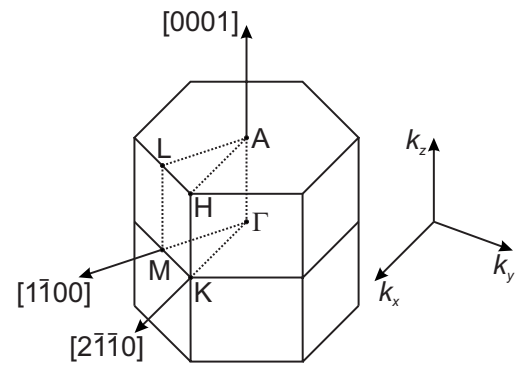

FIG. 1. Hexagonal BZ with high-symmetry points and crystallographic directions (left-hand side) and orientation of $\mathbf{k}$-space directions with respect to the $\mathrm{BZ}$ (right-hand side). High-symmetry directions are indicated by dotted lines; the $\Gamma A$ direction is parallel to the [0001] direction of the crystal. The $k_{z}$ axis is parallel to $\Gamma A$, which is along normal emission for PE from a (0001)-oriented hcp crystal. 
strongly correlated $4 f$ system in the framework of density functional theory (DFT) have been applied mostly to lanthanide compounds using, e.g., self-interaction corrections ${ }^{28}$ or the so-called LSDA $+U$ method $^{29,30}$ or more involved schemes. ${ }^{31}$

Even though there is no a priori justification to identify the band structure calculated with DFT with the results of angle-resolved PE, a detailed comparison between theory and experiment yields important information on the validity of this procedure that has been commonly used in many studies. Such a comparison has been made for some highsymmetry points and directions of Gd (Refs. 7 and 8) and $\mathrm{Tb},{ }^{19}$ while the present work covers all occupied bands within $1.6 \mathrm{eV}$ from the Fermi level for almost all highsymmetry directions. Furthermore, many aspects of magnetism of the heavy lanthanide metals, in particular those connected with helical AFM ordering, are still the subject of both experimental and theoretical studies, ${ }^{22,32}$ where the present results are expected to provide helpful insights.

Band-structure calculations for $\mathrm{Tb}$ metal are much more challenging than those for $\mathrm{Gd}$ metal. The reason is that $\mathrm{Tb}$, with eight $4 f$ electrons in the metallic state, has a nonspherical $4 f$ shell giving rise to a large magnetocrystalline anisotropy, while in Gd, with its half-filled $4 f$ shell, the magnetocrystalline anisotropy is very small. This is a consequence of a large difference in spin-orbit coupling, which must be properly taken into account.

For Tb metal, only a few PE studies of the electronic structure have been performed to date. In Refs. 18 and 19, the dispersions of the $\Delta_{1}$ and $\Delta_{2}$ bands at room temperature were mapped along the $\Gamma A$ direction. In the work of Ref. 18, a bulk $\mathrm{Tb}(0001)$ single crystal was studied, with an Fe contamination on the surface that caused shifts of the valenceband energies towards lower $\left(E-E_{F}\right)$ values, i.e., to higher binding energies. Later on, the study was repeated with a bulk $\mathrm{Tb}$ single crystal free of $\mathrm{Fe}$ contaminations. ${ }^{19}$

In the present work, we report on a detailed and systematic study of the bulk band structure of ferromagnetic $\mathrm{Tb}$ metal at a temperature of $25 \mathrm{~K}$ by means of angle-resolved photoelectron spectroscopy (ARPES). The sample studied was a single-crystalline 100 - $\AA$-thick Tb-metal film grown on a W(110) substrate. In addition, full-potential-linearizedaugmented-plane-wave (FLAPW) calculations were carried out for comparison. We compare the electronic structure particularly in high-symmetry directions of the hexagonal BZ, and list critical points, which represent the energies of the bands at high-symmetry points of the BZ, as well as Fermi vectors in high-symmetry directions; the latter connect $\Gamma$ with points in $\mathbf{k}$ space where bands cross the Fermi level. We also present both experimental and theoretical Fermi-surface contours for the $\Gamma M K$ and the $\Gamma A L M$ planes.

\section{EXPERIMENTAL AND THEORETICAL PROCEDURES}

Clean 100- $\AA$-thick Tb-metal films were grown on a $\mathrm{W}(110)$ substrate by metal vapor deposition at room temperature in a vacuum of $\simeq 5 \times 10^{-10} \mathrm{mbar}$, and subsequently annealed at $700{ }^{\circ} \mathrm{C}$. In this way, well-ordered films with a smooth (0001) surface were obtained. ${ }^{33}$ The sample was transferred in ultrahigh vacuum (UHV) to the analyzer chamber (base pressure $\simeq 1 \times 10^{-11}$ mbar) and mounted on a liquid-He-cooled goniometer, capable of varying by stepmotor-controlled motion two orthogonal polar angles as well as the sample rotation with respect to normal emission. The sample was cooled down to $25 \mathrm{~K}$ without applying an external magnetic field, resulting in a multidomain state, where the magnetization direction is along an easy axis of Tb (hexagonal $\mathbf{b}$ axis). We expect that there is no difference in the spin-integrated PE results for the multidomain samples studied here and a single-domain sample.

Angle-resolved PE spectra were taken with a display-type photoelectron analyzer (Gammadata SES100) and a totalsystem resolution of $\simeq 60 \mathrm{meV}$ (full width at half-maximum) in the photon-energy region from 80 to $200 \mathrm{eV}$ at undulator beamline 7.0.1 of the Advanced Light Source (ALS) at Lawrence Berkeley National Laboratory. The data were uniformly sampled in the three-dimensional space spanned by the photon energy and two polar angles; they were subsequently mapped to $\mathbf{k}$ space using simple trigonometric transformations. Slices along high-symmetry directions of the hexagonal BZ were extracted to obtain band maps at constant $k_{z}$. Additionally, subsets for $E=E_{F}$ with variable k reveal the shape of the FS. The experimental locations of high-symmetry points were obtained by analyzing the periodicities and symmetries of the measured Fermi contours and the electronic structure.

The orientations of the directions in $\mathbf{k}$ space are as follows: The $k_{z}$ direction is parallel to normal emission (see Fig. $1)$, which is parallel to the $\Gamma A$ direction for a (0001)-oriented hcp crystal. The $k_{x}$ direction is parallel to $\Gamma K$ and the $k_{y}$ direction is parallel to $\Gamma M$.

For the bulk band-structure calculations, we employed DFT in the generalized gradient approximation of Perdew et $a l .{ }^{34}$ We use the FLAPW method, ${ }^{35,36}$ as realized in the FLEUR code. The lattice constants were chosen as $a$ $=3.60 \AA$, with a $c / a$ ratio of 1.581 , and the muffin-tin radii were set to $R_{\mathrm{MT}}=2.80$ a.u. The plane-wave cutoff for the basis functions was $K_{\max }=3.4$ a.u. $^{-1}$, and the irreducible part of the BZ was sampled at $128 \mathbf{k}$ points. Spin-orbit coupling was included in the self-consistent calculations as described in Ref. 37. The spin-quantization axis was chosen to be par-

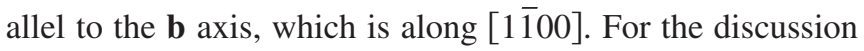
of the bands, we keep the nonrelativistic notation for symmetry and distinguish majority and minority bands, although relativistic effects were included in the calculations. The $5 \mathrm{~s}$ and $5 p$ semicore states were treated as local orbitals, ${ }^{38}$ while the $4 f$ states were included in the core (open core-shell treatment of the $4 f$ electrons). For comparison, we also performed LSDA $+U$ calculations, ${ }^{29}$ with the $4 f$ states included as valence electrons. The occupancy of the minority $4 f$ orbitals was chosen to yield an in-plane orbital moment of almost $3 \mu_{B}$, in line with Hund's rule. We used values of $U$ $=5.7 \mathrm{eV}$ and $J=0.6 \mathrm{eV}$ and this led to a very localized minority $4 f$ state at $-2.9 \mathrm{eV}$. A comparison with the results of our calculations, where the $4 f$ states were included in the core, revealed almost no change in the energies of the valence states in the window of experimental observation. 


\section{RESULTS AND DISCUSSION}

The occupied part of the band structure of $\mathrm{Tb}$ metal consists of four bands-as for other trivalent hcp rare-earth metals - that are filled with one $5 d$ and two $6 s$ electrons. In the ferromagnetic state, these four bands are exchange split and cover the energy region from $-5.32 \mathrm{eV}$ to $2.34 \mathrm{eV}$ relative to the Fermi energy. All four bands cross the Fermi energy at various positions in $\mathbf{k}$ space, forming a much more complex FS than in simple or noble metals like, e.g., $\mathrm{Cu}$, where the FSs are almost spherical free-electron-like. In the free-electron case, all Fermi vectors, which are defined as the wave vectors $\mathbf{k}=\left(k_{x}, k_{y}, k_{z}\right)$ of those electronic states that are located at the Fermi energy, have the same absolute value $\left|\mathbf{k}_{F}\right|$. In Tb metal, the $E_{F}$ crossings of the various bands occur at Fermi vectors with different $\left|\mathbf{k}_{F}\right|$; the Fermi vectors of Tb metal in high-symmetry directions will be listed further below.

Before we go into details of the electronic structure of ferromagnetic $\mathrm{Tb}$ metal, we shall briefly discuss a general aspect of PE on hcp crystals, specifically the seemingly doubled $\mathrm{BZ}$ dimensions in the $\Gamma A$ direction as compared to theoretical considerations, and we shall show how this can be derived from symmetry properties of the hcp crystal in combination with dipole selection rules. To describe the periodicity of the hcp lattice, one needs a two-atom basis, with the second atom representing the crystal plane at $c / 2$, where $c$ represents the hexagonal lattice constant in the [0001] direction. Since both lattices, the hcp and the fcc, consist of close-packed planes, their symmetries and electronic structures are related. The hcp primitive translation in the [0001] direction, however, is 2 times as long as the one in the fcc [111] direction, assuming identical NN spacings. This doubled primitive translation in hcp real space corresponds to a halving of the distance between two $\Gamma$ points in the hcp k space (along the hcp $\Gamma A \Gamma$ and the fcc $\Gamma L \Gamma$ directions, respectively). This introduces a BZ boundary, where electronic bands propagating in the $k_{z}$ direction are backfolded. Due to the presence of symmetry elements with nonsymmorphic translations, at this zone boundary the backfolded bands remain degenerate. Spin-orbit coupling can lift this degeneracy, but the effect is rather small. ${ }^{39}$ Due to the similarities between the hcp and the fcc lattice, hcp bands can be referred to as fcc $\Gamma$-like or fcc $L$-like, depending on whether they are closer to an fcc $\Gamma$ point or to an fcc $L$ point, respectively; ${ }^{40}$ alternatively, they can be referred to as $\Delta_{1}$-like and $\Delta_{2}$-like in hcp terms.

PE is capable of distinguishing between these two symmetries by adjusting the photon energy, which in turn changes the momentum of the photoelectron. To reach the (0001) surface and eventually the detector, the photoelectron must have a plane-wave component parallel to the surface normal. As shown in Ref. 41, such a plane wave $\mathbf{k}$ $=\left(0,0, k_{z}\right)$ propagating in an hcp lattice, where $k_{z}$ is the component along the [0001] direction, originates from bands with either $\Delta_{1}$ or $\Delta_{2}$ symmetry, depending on $|\mathbf{k}|$. This symmetry is preserved in the PE process, as one can see from the dipole selection rules for hcp crystals. ${ }^{42}$

In the region of photon energies employed in this study, one must take into account the $\mathrm{Tb} 4 d \rightarrow 4 f$ giant resonance (between about $130 \mathrm{eV}$ and $180 \mathrm{eV}$ ), which causes a strong variation of the $4 f \mathrm{PE}$ cross section due to resonant photoemission. ${ }^{43}$ The reason is that the final states of $\mathrm{Tb}$ excited by direct PE can also be reached by autoionization decay channels of the $4 d^{9} 4 f^{9}$ intermediate state excited in the giant resonance process from the $4 d^{10} 4 f^{8}$ initial state of $\mathrm{Tb}$. The dominant autoionization decay channel is the rapid super-Coster-Kronig decay, which leads to a $4 d^{10} 4 f^{7}$ final state, such as in direct $4 f \mathrm{PE}$. $^{44}$ Other autoionization decay channels influence the PE intensities from valence states and consequently the PE spectra from band states, in particular when their dispersion is studied perpendicular to the surface by variation of the photon energy.

\section{A. Band dispersions}

In the following, band dispersions along high-symmetry directions of the hexagonal BZ will be presented, with a comparison of the experimental and theoretical results particularly for the $\Gamma M K$ and the $A L H$ planes. For the $\Gamma A, M L$, and $K H$ directions, only calculated bands will be shown, due to experimental difficulties arising mainly from small PE cross sections of the bands and substantial PE intensity variations caused by resonant PE when the photon energy is scanned through the $\mathrm{Tb} 4 d \rightarrow 4 f$ giant resonance around $h \nu$ $=150 \mathrm{eV}$.

Figure 2 shows a comparison between (a),(b) the measured electronic structure of $\mathrm{Tb}$ metal at $25 \mathrm{~K}$ and (c) the theoretical results for the $\Gamma M K$ plane of the BZ (see Fig. 1). The top panels in (a) and (b) display the as-measured data, where dark gray corresponds to high PE intensities. For clarity, the experimental positions of the bands are shown in the bottom panels in (a) and (b) as guides to the eyes. As discussed, PE is capable of distinguishing between the two different symmetry types of bands by choosing the appropriate photon energy. The $\Delta_{2}$-like bands 2 and 3, shown in Fig. 2(a), were observed at a photon energy of $\simeq 105 \mathrm{eV}$; they correspond to the bands colored red in Fig. 2(c). The $\Delta_{1}$-like bands 1 and 4, shown in Fig. 2(b), were observed at a photon energy of $\simeq 155 \mathrm{eV}$, and they correspond to the blue bands in (c).

Figure 2(a) displays the majority $(\uparrow)$ and minority $(\downarrow)$ bands 2 and 3 , both with $\Delta_{2}$-like symmetry. Band 2 is exchange split by $E_{\mathrm{ex}} \simeq 0.9 \mathrm{eV}$ (where $E_{\mathrm{ex}}$ depends on k), and starting at $\Gamma$-where only the minority band is within the detector window-both subbands $2 \uparrow$ and $2 \downarrow$ disperse upward. When approaching $M$, their slopes change sign and the PE intensity decreases. Figure 2(a) displays also the dispersions of the exchange-split bands $3 \uparrow$ and $3 \downarrow$, which at $\Gamma$ are both located above $E_{F}$; toward $M$ and $K$, they both disperse downwards, creating a hole pocket of the Tb Fermi surface. For critical points and Fermi vectors, see Tables I and II. Minority bands are observed with higher PE peak intensities than their majority counterparts, an observation that can be attributed to different lifetime broadenings. This can be best seen from the valence-band spectrum at half-way $\Gamma M$ shown in Fig. 5(b). Its position on the $\Gamma M$ line is indicated by the arrow in the top panel of Fig. 2(a). In Refs. 45 and 46, the scattering mechanisms that are relevant for the lifetimes of 

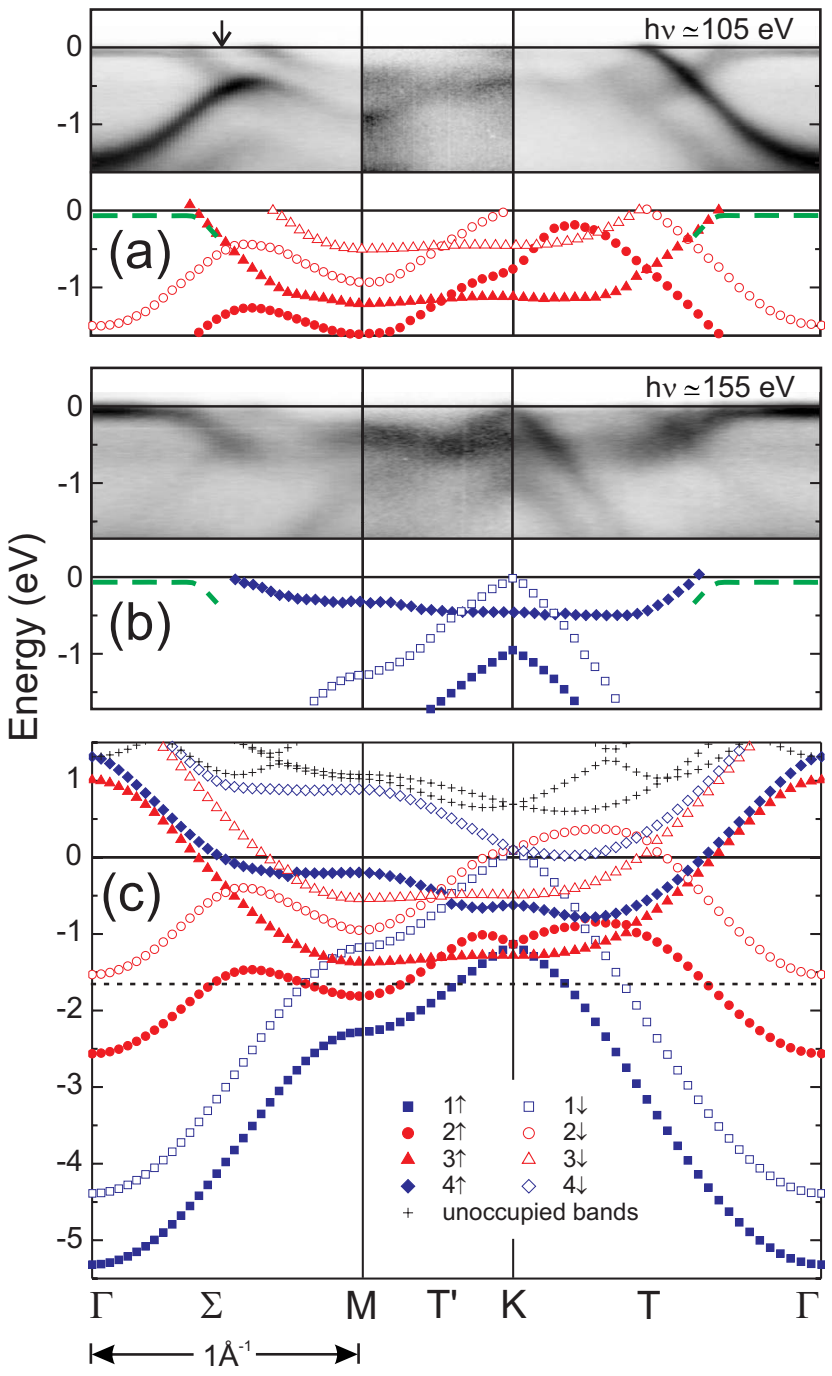

FIG. 2. (Color) Comparison of (a),(b) the experimental electronic structure of $\mathrm{Tb}$ metal at $25 \mathrm{~K}$ with (c) the results of our calculations for the $\Gamma M, M K$, and $K \Gamma$ high-symmetry lines. The top panels in (a) and (b) show the PE data as measured. Dark gray tones represent high PE intensity; note that the gray levels in the sections $\Gamma M, M K$, and $K \Gamma$ are scaled individually, to compensate for matrixelement effects. The bottom panels serve as guides to the eye and reflect the experimental positions of the bands [same symbols as in (c)]. PE can distinguish between (a) states with $\Delta_{2}$-like symmetry at $h \nu \simeq 105 \mathrm{eV}$ and (b) states with $\Delta_{1}$-like symmetry at $h \nu \simeq 155 \mathrm{eV}$ (for details, see text). The majority part of the $d$-like surface state is clearly visible in the symmetry band gap around $\Gamma$ both in (a) and (b) (green dashed lines in the bottom panels), reflecting a good quality of the sample. The calculated bands in (c) are in very good agreement with the experimental results in (a),(b). The surface state is absent in the bulk calculations. Red color corresponds to $\Delta_{2}$-like (bands 2 and 3) and blue color to $\Delta_{1}$-like symmetry (bands 1 and 4), filled (open) symbols represent majority (minority) electronic states. Bands that are not involved in the formation of the Fermi surface are represented by plus symbols $(+)$. The area between $E_{F}$ and the short-dashed line in (c) marks the experimental window.
TABLE I. Energies of critical points of the electronic structure of $\mathrm{Tb}$ metal relative to $E_{F}$ (in $\mathrm{eV}$ ); the experimental error bars are estimated to be $\pm 50 \mathrm{meV}$. The nonrelativistic notation of the critical points is according to Refs. 1 and 53.

\begin{tabular}{|c|c|c|c|c|c|c|}
\hline Band 1 & $\Gamma_{1}^{+} \uparrow$ & $\Gamma_{1}^{+} \downarrow$ & $M_{1}^{+} \uparrow$ & $M_{1}^{+} \downarrow$ & $K_{5} \uparrow$ & $K_{5} \downarrow$ \\
\hline Expt. & & & & -1.20 & -0.94 & $0.00^{\mathrm{a}}$ \\
\hline Theor. & -5.32 & -4.39 & -2.28 & -1.18 & -1.13 & 0.10 \\
\hline Band 2 & $\Gamma_{4}^{-\uparrow}$ & $\Gamma_{4}^{-} \downarrow$ & $M_{2}^{-} \uparrow$ & $M_{2}^{-} \downarrow$ & $K_{5} \uparrow$ & $K_{5} \downarrow$ \\
\hline Expt. & & -1.50 & $-1.60^{\mathrm{b}}$ & -0.89 & $-0.89^{c}$ & $0.00^{\mathrm{a}}$ \\
\hline Theor. & -2.56 & -1.53 & -1.81 & -0.95 & -1.13 & 0.10 \\
\hline Band 3 & $\Gamma_{3}^{+} \uparrow$ & $\Gamma_{3}^{+} \downarrow$ & $M_{3}^{+} \uparrow$ & $M_{3}^{+} \downarrow$ & $K_{2} \uparrow$ & $K_{2} \downarrow$ \\
\hline Expt. & & & $-1.20^{\mathrm{c}}$ & -0.50 & -1.07 & -0.48 \\
\hline Theor. & 1.01 & 1.96 & -1.37 & -0.54 & -1.28 & -0.49 \\
\hline Band 4 & $\Gamma_{6}^{-\uparrow}$ & $\Gamma_{6}^{-} \downarrow$ & $M_{1}^{+\uparrow}$ & $M_{1}^{+} \downarrow$ & $K_{1} \uparrow$ & $K_{1} \downarrow$ \\
\hline Expt. & & & -0.41 & & -0.46 & \\
\hline Theor. & 1.31 & 2.34 & -0.20 & 0.89 & -0.62 & 0.10 \\
\hline Band 1/2 & $A_{1} \uparrow$ & $A_{1} \downarrow$ & $L_{1} \uparrow$ & $L_{1} \downarrow$ & $H_{2} \uparrow$ & $H_{2} \downarrow$ \\
\hline Expt. & & & & -1.02 & -0.46 & \\
\hline Theor. & -3.76 & -2.79 & -2.28 & -1.42 & -0.98 & 0.03 \\
\hline Band $3 / 4$ & $A_{1} \uparrow$ & $A_{1} \downarrow$ & $L_{1} \uparrow$ & $L_{1} \downarrow$ & $H_{1} \uparrow$ & $H_{1} \downarrow$ \\
\hline Expt. & & & -0.47 & & $-0.76^{\mathrm{c}}$ & \\
\hline Theor. & 0.52 & 1.60 & -0.48 & 0.64 & -0.68 & 0.07 \\
\hline
\end{tabular}

a Bands cross $E_{F}$ in the vicinity of the $K$ point, which makes a precise determination of their energies difficult in PE experiments. ${ }^{\mathrm{b}}$ Critical point at lower border of the experimental window $(-1.6 \mathrm{eV})$; the experimental error bar is therefore larger.

${ }^{\mathrm{c}}$ Larger experimental error bar due to multiple band crossings.

photoholes-i.e., electron-electron, electron-phonon, and electron-magnon scattering-are discussed for the case of the Gd (0001) surface state.

In the projected band gap around $\Gamma$, the majority part of the $d$-like (0001) surface state is observed, reflected by the green dashed lines in the bottom panel, located at an energy of $-0.07 \pm 0.05 \mathrm{eV}$. Away from $\Gamma$, it meets band $3 \uparrow$ and forms a surface resonance. The presence of the surface state reflects good crystallinity of the sample with a flat and clean surface. The minority surface state is above $E_{F}$ and is accessible, e.g., by inverse photoemission ${ }^{47}$ or by scanningtunneling spectroscopy. ${ }^{46}$

Figure 2(b) shows the $\Delta_{1}$-like subbands $1 \uparrow$ and $1 \downarrow$ as well as $4 \uparrow\left(4 \downarrow\right.$ is located above $\left.E_{F}\right)$, together with the majority surface state and the surface resonance. Due to the $4 d \rightarrow 4 f$ $\mathrm{PE}$ resonance at $h \nu \simeq 150 \mathrm{eV}$, the surface state intensity is enhanced, as compared to the data shown in (a). Band $4 \uparrow$ can be traced between its crossing with $E_{F}$ at half-way along $\Gamma M$ and its second crossing with $E_{F}$ along $K \Gamma$. Analogous to $3 \uparrow$ and $3 \downarrow$, it forms also a hole pocket on the Tb Fermi surface. Close to $M$ in the $\Gamma M$ section, $1 \downarrow$ appears in the detector window, dispersing upward from $M$ to $E_{F}$ in the vicinity of 
TABLE II. Fermi vectors (in $\AA^{-1}$ ) in high-symmetry directions of the $\mathrm{BZ}$ of $\mathrm{Tb}$ metal (see text); the experimental error bars are $\pm 0.03 \AA^{-1}$ (no band crossings with $E_{F}$ in the $\Gamma A$ direction).

\begin{tabular}{lcccc}
\hline \hline Section & Distance & Band & Expt. & Theor. \\
\hline$\Gamma M$ & 1.01 & $3 \uparrow$ & 0.37 & 0.39 \\
& & $4 \uparrow$ & 0.52 & 0.48 \\
& & $3 \downarrow$ & 0.66 & 0.65 \\
$M K$ & 0.58 & $2 \downarrow$ & 0.58 & 0.47 \\
& & $1 \downarrow$ & 0.58 & 0.54 \\
$\Gamma K$ & 1.16 & $3 \uparrow$ & 0.37 & 0.41 \\
& & $4 \uparrow$ & 0.50 & 0.45 \\
& & $2 \downarrow$ & 0.65 & 0.60 \\
& & $3 \downarrow$ & 0.65 & 0.69 \\
$A L$ & $1 \downarrow$ & 1.15 & 1.13 \\
$L H$ & 1.01 & $3 / 4 \uparrow$ & 0.51 & 0.36 \\
$A H$ & 0.58 & $1 / 2 \downarrow$ & 0.47 & 0.57 \\
& 1.16 & $3 / 4 \uparrow$ & 0.50 & 0.39 \\
$K H$ & & $1 / 2 \downarrow$ & 1.09 & 1.15 \\
$M L$ & 0.55 & $3 \downarrow$ & & 0.46 \\
\hline \hline
\end{tabular}

$K$. Around $K, 1 \uparrow$ is observed as well, with both subbands dispersing downwards and out of range along $K \Gamma$. Figure 2(b) also contains traces of the most intense bands with $\Delta_{2}$-like symmetry, i.e., $2 \uparrow$ and $2 \downarrow$ [see Fig. 2(a)]. We attribute their appearance to the $4 d \rightarrow 4 f$ giant resonance, which allows for additional excitation channels (e.g., Auger excitation) that are not governed by dipole selection rules. Moreover, a blurring of the $k_{z}$ component introduced by surface defects as well as a weakening of the dipole selection rules by relativistic effects are possible causes.

The theoretical electronic structure calculated in this work exhibits a remarkably good agreement with the experimental results from the PE study, as can be seen from Fig. 2(c). However, a few details-like some critical points and Fermi vectors-are at variance, as will be discussed further below. To classify the bands according to symmetry and spin, we performed additional scalar-relativistic calculations (not shown here) and assigned the results, wherever possible, to bands of the calculation, where spin-orbit coupling was included; although some band crossings are lifted due to spinorbit coupling effects, this procedure can be applied unambiguously to all bands. Here, only at least partially occupied bands and their exchange-split counterparts are colored (blue for $\Delta_{1}$-like and red for $\Delta_{2}$-like symmetry); filled (open) symbols represent majority (minority) bands. The exchange splitting of the bands is found to vary between 0.7 and $1.0 \mathrm{eV}$. This can be compared with the calculated magnetic moment of the delocalized band states of $0.70 \mu_{B}$. A calculation of the magnetic moments of the $4 f$ states included in the valence window (using an LSDA $+U$ calculation) shows that these states contribute almost exactly $6.0 \mu_{B}$ to the spin moment and 3.0 $\mu_{B}$ to the orbital moment per Tb atom. A comparison with the results of calculations for Gd (Ref. 10) shows that the spin polarization of the band states is approximately pro-

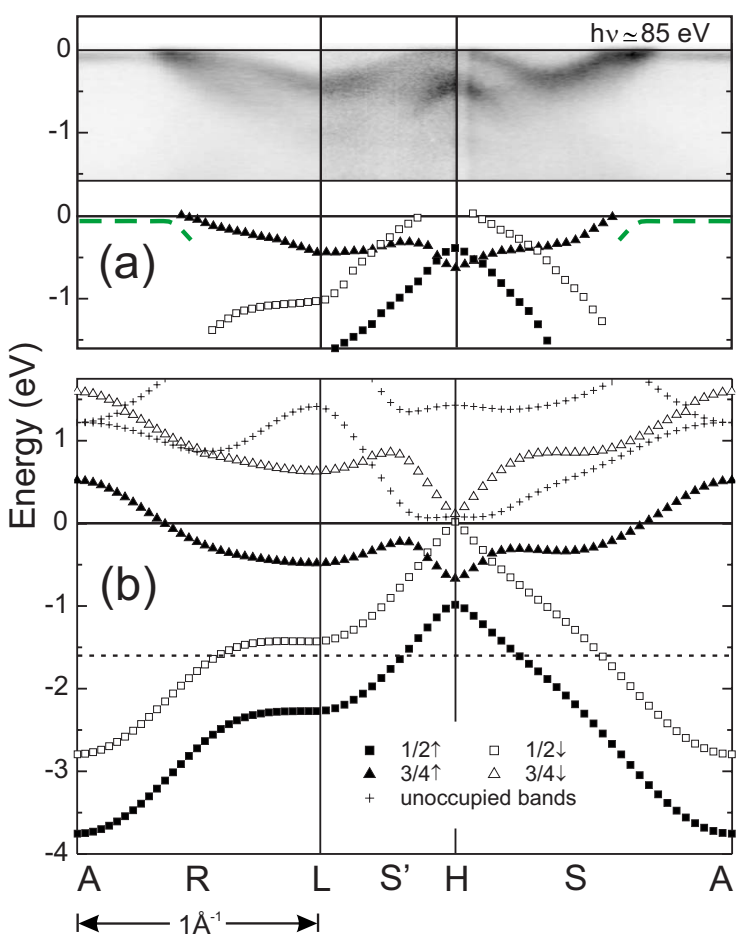

FIG. 3. (Color online) Comparison of (a) the experimental banddispersion curves measured at $h \nu \simeq 85 \mathrm{eV}$ with (b) the results of theory in $A L, L H$, and $H A$ high-symmetry directions. Bands $1 / 2$ and $3 / 4$ are degenerate due to backfolding at the $A L H$ plane. The top panel in (a) shows the data as measured, where dark gray tones correspond to high PE intensities. The bottom panel in (a) displays the experimental band positions as guides to the eye; the same notation as in (b) is used. Green (gray) dashed lines indicate the location of the surface state and surface resonance (see text). In (b) filled (open) symbols represent majority (minority) bands; electronic states above $E_{F}$ are marked with a plus symbol (+). The area between $E_{F}$ and the dashed line reflects the experimental window.

portional to the $4 f$ spin moment. This complies with the experimental observation that the magnetic exchange splitting of surface states on the rare-earth metals is also approximately proportional to the $4 f$ spin moment. ${ }^{46}$ Experimentally, a saturation magnetization of $9.34 \mu_{B}$ was determined for $\mathrm{Tb}$ metal, ${ }^{48}$ which is - like in $\mathrm{Gd}$ - slightly smaller than the calculated value $\left(9.7 \mu_{B}\right)$.

Figure 3(a) displays the experimental electronic structure, obtained at $h \nu \simeq 85 \mathrm{eV}$ in $A L, L H$, and $H A$ directions. Green (gray) dashed lines in the $A L$ and $H A$ band maps on the bottom panel of (a) indicate the majority part of the $d$-like (0001) surface state and the surface resonance with band $3 \uparrow$. It is located at the $\left(k_{x}, k_{y}\right)$ positions of band $3 \uparrow$ in the $\Gamma M K$ plane and is visible in the $A L H$ plane due to the indefinite $k_{z}$ component that is caused by its confinement to the surface layer. The bottom panel of Fig. 3(a) reflects the experimental band positions as guides to the eyes. Since bands are backfolded at the $A L H \mathrm{BZ}$ boundary (resulting in a change from $\Delta_{1}$ to $\Delta_{2}$ symmetry, and vice versa) without the formation of band gaps, all electronic states are twofold degenerate, resulting in bands $1 / 2$ and $3 / 4$, which are located at the same positions in $\mathbf{k}$ space. Starting above $E_{F}$ at $A$, band $3 / 4 \uparrow$ 
moves below the Fermi energy at about half-way along $A L$ (on the $L$ side of the high-PE-intensity section at $E_{F}$, which is caused by the surface resonance), and it stays below $E_{F}$ along $L H$; it crosses $E_{F}$ again at half-way along $H A$. Also visible in the experimental dispersion curves in Fig. 3(a) is band $1 / 2 \downarrow$, which crosses $E_{F}$ close to $H$, as well as band $1 / 2 \uparrow$, which is at $-0.46 \mathrm{eV}$ at $\mathrm{H}$. The theoretical results shown in (b) reflect the characteristics of $3 / 4 \uparrow$ accurately, whereas $1 / 2 \uparrow$ and $1 / 2 \downarrow$ are located at too low energies, and their magnetic exchange splitting is overestimated by about $250 \mathrm{meV}$ at $H$. In general, the experimentally observed behavior of the electronic states on the $A L H$ plane is not as accurately reproduced by the theoretical results as on the $\Gamma$ $M K$ plane.

In PE experiments, it is difficult to map out the dispersions of valence bands perpendicular to the surface, i.e., in our case perpendicular to $\Gamma M, M K$, and $\Gamma K$, due to the spread in $k_{\perp}$ implied by finite lifetimes of the final states, or correspondingly, in the space domain, due to short electron mean-free paths. ${ }^{49}$ The momentum broadening [full width at half-maximum (FWHM)] $\Delta k_{f \perp}=1 / l(E),{ }^{50}$ where $l(E)$ denotes the energy-dependent electron mean-free path, can be roughly estimated from the universal curve, ${ }^{51}$ which gives $l$ $\simeq 10 \AA$ for energies around $150 \mathrm{eV}$, resulting in $\Delta k_{f \perp}$ $\simeq 0.1 \AA^{-1}$. In Ref. 52, the connection between the measured lifetime width $\Gamma_{m}$ and the lifetime width of the photoelectron $\Gamma_{f}$ as well as that of the photohole $\Gamma_{i}$ has been discussed for the three different principal modes of PE, namely energy distribution curve (EDC), constant-initial-state spectroscopy (CIS), and constant-final-state spectroscopy (CFS). To this end, the group velocities $v_{f \perp}\left(\hbar v_{f \perp}=\partial E_{f} / \partial k_{\perp}\right)$ and $v_{i \perp}$ for a given system must be considered. In CIS, $E_{i}$ is kept constant, and the spectrum is recorded by sweeping the kinetic energy of the photoelectron and the photon energy simultaneously; this results in $\Gamma_{m}=\left|v_{f \perp} / v_{i \perp}\right| \Gamma_{i}+\Gamma_{f}$ (at normal emission). ${ }^{52}$ Thus, for tracing band dispersions perpendicular to the surface, $\Delta k_{f \perp}=0.1 \AA^{-1}$ (FWHM) can be considered as a lower limit for momentum broadening perpendicular to the surface. In addition, in the $\mathrm{Tb}$ case, a measurement of band dispersion maps perpendicular to the surface is complicated by the atomic $4 d \rightarrow 4 f$ giant resonance at photon energies of $h \nu$ $\simeq 150 \mathrm{eV}$, which causes substantial changes of the PE intensity due to resonant PE already at minor variations of the photon energy as well as large background effects. Since the bands along $K H$ and $M L$ are characterized by small PE cross sections, they turn out to be rather difficult to trace. For these reasons, experimental data for these sections are not shown here. Along $\Gamma A$, no band is within the detector window, which covers the energy region from $-1.6 \mathrm{eV} \leqslant\left(E-E_{F}\right)$ $\leqslant 0 \mathrm{eV}$.

Bands 1,2 and 3,4, which are well separated in energy on the $\Gamma M K$ plane of the BZ, disperse toward each other in the direction perpendicular to the surface, and meet in the $A L H$ plane, as can be seen in Fig. 4 for (a) the $\Gamma A$, (b) the $K H$, and (c) the $M L$ direction. Outside $A L H$, only $1 / 2 \uparrow$ and $1 / 2 \downarrow$ are degenerate along $K H$. The absence of band gaps in connection with the backfolding at the $A L H$ plane can be understood from symmetry arguments. ${ }^{39}$ Along the line $A L$, this degeneracy is even present when spin-orbit coupling is included, at other points on the $A H L$ plane the splitting is too small to be seen in the plots. The calculated non-spinpolarized band structure along the line $\Gamma A$ has been compared with experimental results in Ref. 19; our spin-split dispersion in this direction is similar in shape as that presented in Ref. 19. A comparison of the spin splitting in theory and experiment can be found in Ref. 8 for Gd. Summarizing these results, reasonable agreement between calculations and measurement was found, so we are confident that our calculations give a good description of future measurements along the $\Gamma A$ line.

\section{B. Critical points and Fermi vectors}

Table I gives a summary of the measured and calculated critical points, which represent the energy positions of the bands at high-symmetry points of the BZ. The experimental results were obtained by fitting the spectra at the corresponding positions in $\mathbf{k}$ space; only critical points within the experimental window, i.e., in the energy region from 0 to $-1.6 \mathrm{eV}$ relative to $E_{F}$, were accessed. Since bands 1 and 2 are degenerate on the $A L H$ plane, data for these two bands are listed together; the same applies for bands 3 and 4 . Comparing the results for the $\Gamma M K$ plane, it is striking that the calculated energies of critical points of the minority states are 10-to- $60 \mathrm{meV}$ below the measured ones, whereas for the majority states, the systematic deviation is about $200 \mathrm{meV}$, also toward lower $\left(E-E_{F}\right)$ values (higher binding energies). This leads to an overestimation of the exchange splitting by about $175 \mathrm{meV}$, which is similar to what has been found for the exchange splitting of the surface state of $\mathrm{Gd} .{ }^{10} \mathrm{On}$ the $A L H$ plane, some critical points show deviations up to $500 \mathrm{meV}$.

Figure 5 shows examples of the least-squares-fit analysis. The band components were described by Lorentzians. Smooth backgrounds were introduced [dashed lines in (a) and (b)] to account for inelastic processes and PE from other regions of the BZ. Good fit results were obtained when the background was kept constant, which is in agreement with low-temperature Tb spectra shown in Ref. 15. During the fit procedure, the subspectra were numerically convolved with a Gaussian to account for experimental resolution. In (a), at the $\Delta_{2}$-like $\Gamma$ point, band $2 \downarrow$ is in the detector window at an energy of $-1.50 \pm 0.05 \mathrm{eV}$. Additional lines were used for the surface state $S$ and a weak background contribution at an energy of $\simeq-0.6 \mathrm{eV}$. We attribute the latter to non-k conserving emission from nearby parts of the BZ, probably originating from band $2 \downarrow$ that disperses upward to $M$ and $K$ with high PE intensities. Half-way along $\Gamma M$, at the position indicated by a vertical arrow in the top panel of Fig. 2(a), both subbands $2 \uparrow$ and $2 \downarrow$ are in the experimental window. In order to quantify their different lifetime broadenings, the corresponding EDC and fit analysis is shown in Fig. 5(b). Both subbands are found to contribute with almost the same PE intensities (amplitudes of the Lorentzians). Bands $2 \uparrow$ and $2 \downarrow$ exhibit Lorentzian widths of about $300 \mathrm{meV}$ and $100 \mathrm{meV}$, respectively, which reflects the larger lifetime broadenings for the majority states [see discussion of Fig. 2(a)].

To derive the experimental Fermi vectors $\mathbf{k}_{F}$, several different methods were applied. The most reliable results were 

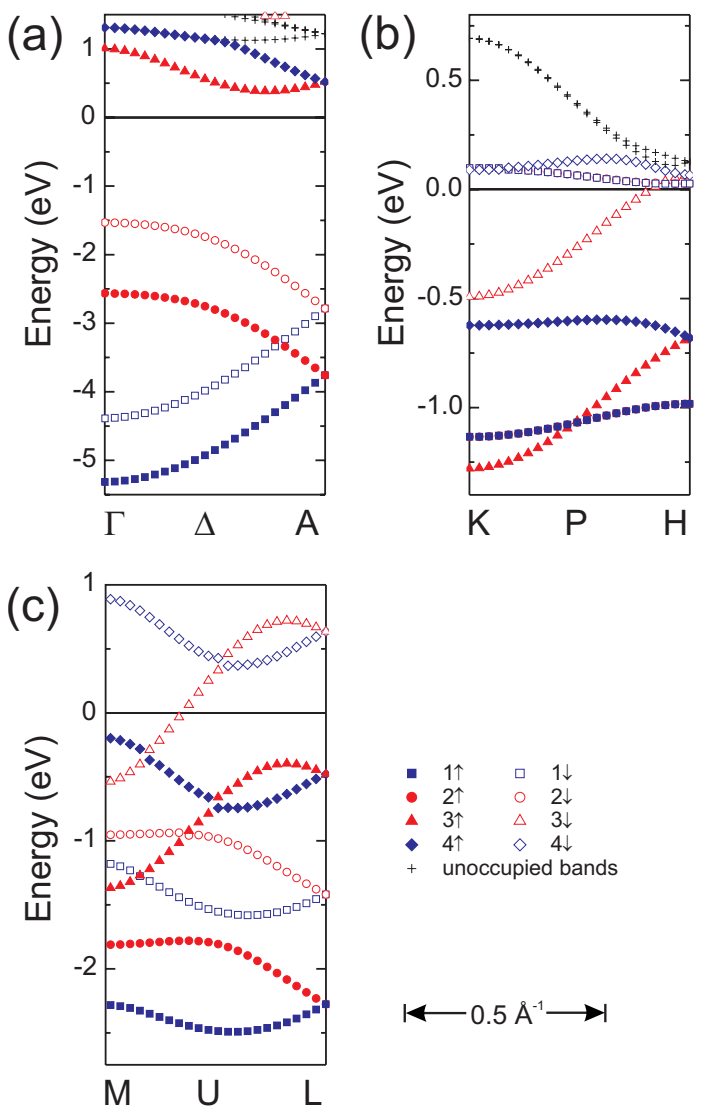

FIG. 4. (Color) Calculated bands in (a) $\Gamma A$, (b) $K H$, and (c) $M L$ high-symmetry directions of the BZ perpendicular to the (0001) surface (note the different energy scales). Bands $1 / 2$ and $3 / 4$ are degenerate on the $A L H$ plane. The degeneracy is lifted when the bands disperse toward the $\Gamma M K$ plane, with the exception of $1 / 2 \uparrow$ and $1 / 2 \downarrow$, which stay degenerate along $K H$. Blue color represents $\Delta_{1}$-like symmetry (bands 1 and 4 ) and red color $\Delta_{2}$-like symmetry (bands 2 and 3); filled (open) symbols represent majority (minority) electronic states. Bands that are not involved in the formation of the Fermi surface are marked with a plus symbol $(+)$.

obtained by a combination of the dispersion method with the maximum intensity method. In the former one, the position of the band in various EDCs for different $\mathbf{k}_{\|}$is extrapolated to $E_{F}$; in the latter one, the $\mathbf{k}_{\|}$position in the momentum distribution curve (MDC) for $E=E_{F}$ that exhibits maximum $\mathrm{PE}$ intensity is considered as $\mathbf{k}_{F}$. These and other commonly accepted techniques for determining Fermi vectors are discussed at length, e.g., in Ref. 54. The application of additional methods to determine $\mathbf{k}_{F}$, e.g., symmetrization of the EDCs with respect to $E_{F}$ or determination of the maximum gradient in the integrated-intensity MDC, where at each $\mathbf{k}_{\|}$ position the corresponding EDC is integrated over the binding energy, did not lead to higher accuracies. The rather accurate $\Delta T$ method, which is based on the assumption of a temperature-independent spectral function $A(\mathbf{k}, \omega),{ }^{55}$ cannot be applied here. The reason is that in a magnetic metal, such as $\mathrm{Tb}, A(\mathbf{k}, \omega)$ changes significantly with temperature due to the shrinking of the magnetic exchange splitting when the material is warmed up. From the above considerations, the

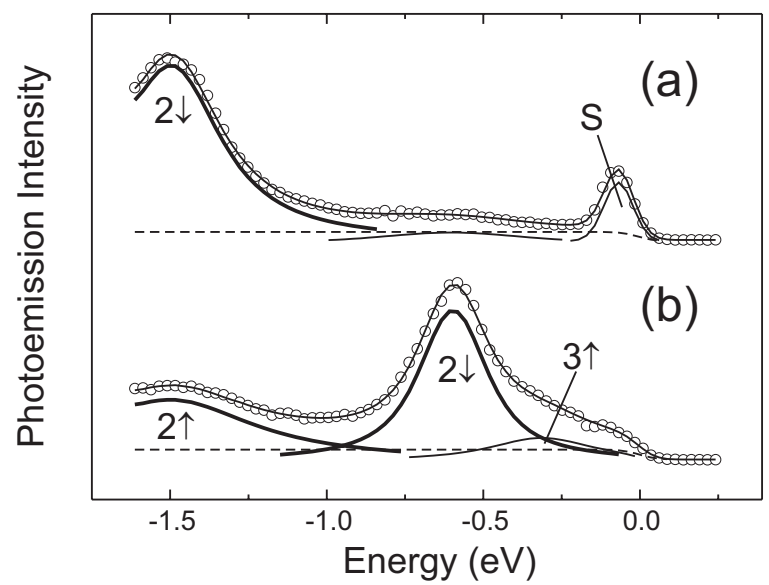

FIG. 5. Valence band spectra of Tb at $T=25 \mathrm{~K}$, measured with $h \nu \simeq 105 \mathrm{eV}$ (a) at the $\Delta_{2}$-like $\Gamma$ point and (b) half-way along $\Gamma M$ [see vertical arrow in Fig. 2(a)]. The solid lines through the data points and the subspectra represent the results of least-squares-fit analyses. In (a), an additional peak $S$ was added to describe the surface state.

experimental error bars for Fermi vectors are estimated to be $\pm 0.03 \AA^{-1}$.

Table II summarizes the BZ dimensions of Tb metal, derived from textbook lattice constants (see, e.g., Ref. 56) transformed to reciprocal space, as well as the Fermi vectors for all bands involved in the formation of the FS of Tb metal. In $\Gamma M, \Gamma K, A L$, and $A H$ directions, the in-plane components of the Fermi vectors $\left|\mathbf{k}_{\|}\right|=\left|\left(k_{x}, k_{y}\right)\right|$ are listed; in $M K, L H$, $K H$, and $M L$ directions, the distances to respective highsymmetry points are given. The table is organized as follows: The first column denotes high-symmetry directions; the second column gives distances between the respective high-

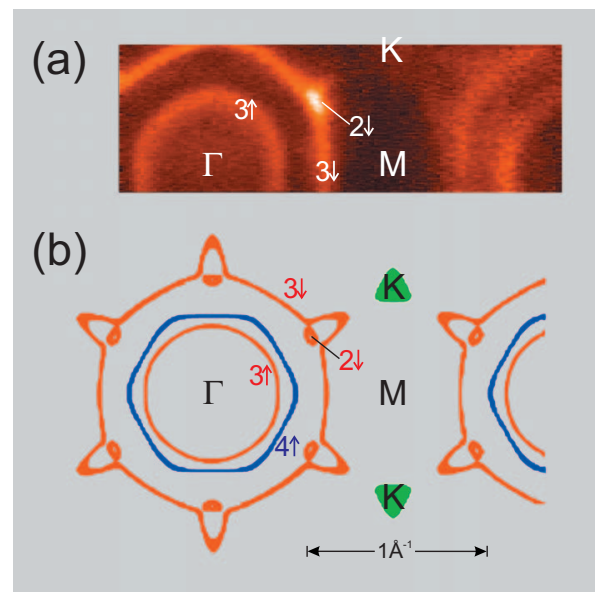

FIG. 6. (Color) Fermi contours in the $\Gamma M K$ plane, i.e., parallel to the sample surface: (a) Experimental data as measured at $h \nu$ $\simeq 105 \mathrm{eV}$, revealing bands 2 and 3 with $\Delta_{2}$-like symmetry [marked in red in (b)]. Bright colors correspond to high PE intensities. (b) Calculated Fermi contours; $\Delta_{1}$-like bands are colored blue, $\Delta_{2}$-like bands are colored red. Around $K$, bands of both symmetries ( $1 \downarrow$ and $2 \downarrow)$ cross $E_{F}$ (marked in green color). The agreement between experiment and theory is excellent. 
symmetry points. The third column lists bands that cross $E_{F}$ along the given high-symmetry directions. The fourth and fifth columns list experimental and theoretical distances, respectively, from the origin of the section to the crossings with the Fermi energy. As an example, in the $\Gamma M$ direction ( $\Gamma M$ distance of $1.01 \AA^{-1}$ ), the bands $3 \uparrow, 4 \uparrow$, and $3 \downarrow$ cross the Fermi energy at experimental distances from $\Gamma$ of $0.37 \AA^{-1}, 0.52 \AA^{-1}$, and $0.66 \AA^{-1}$, respectively; in the $M K$ direction, the bands $2 \downarrow$ and $1 \downarrow$ cross $E_{F}$ both at a distance of $0.58 \AA^{-1}$ from $M$ (i.e., at $\mathrm{K}$ ), etc. The corresponding theoretical values calculated in this work are listed in the fifth column. Note that in $\Gamma A$ direction, there is no band that crosses the Fermi energy. Due to weak PE cross sections along $M L$ and $K H$, together with drastic variations in the PE intensity, when the photon energy is changed ( $\mathrm{Tb} 4 d \rightarrow 4 f$ giant resonance), no experimental Fermi vectors along these directions are given. In $\Gamma M, M K$, and $\Gamma K$ high-symmetry directions, the agreement between experiment and theory is very good, well within the experimental error bars (except for band $2 \downarrow$ and $4 \uparrow$ ). Along $A L, L H$, and $A H$ directions, the experimental error bars cannot account for the differences between the calculated and the measured values. It should be noted that the calculations of Ahuja et al. ${ }^{21}$ give slightly better Fermi vectors along the $A H$ and $A L$ lines, but at the expense of worse agreement in the $\Gamma M K$ plane. Since no details of these calculations were given in Ref. 21, it is difficult to identify the source of the differences in the two theoretical approaches.

\section{Fermi contours}

For several decades, PE has been the primary tool for studies of the electronic structure and FSs. Initially, nondisplay-type electron spectrometers were used to record a great number of EDCs at different angles (and photon energies), making Fermi-surface mapping a tedious procedure (see, e.g., Ref. 57). The situation changed dramatically when display-type electron spectrometers were introduced. Santoni et al., e.g., have directly measured two-dimensional cuts through the Fermi surface of graphite for different photon energies by using a two-angle display-type $\mathrm{PE}$ spectrometer. ${ }^{58}$ Momentum distribution curves for $E=E_{F}$, where high PE intensities indicate band crossings with the Fermi level, are still commonly in use.

Figure 6 displays experimental and theoretical cuts through the Fermi surface of $\mathrm{Tb}$ metal by the $\Gamma M K$ plane. The experimental data [Fig. 6(a)] represents the PE intensity for $E=E_{F}$ measured at $h \nu \simeq 105 \mathrm{eV}\left(\Delta_{2}\right.$-like symmetry $)$, with bright colors corresponding to high PE intensity. This means that the bright-colored contours in Fig. 6(a) represent MDCs for $E=E_{F}$, i.e., Fermi contours. They were obtained from individual ARPES data sets recorded with various photon energies for different tilt angles of the sample surface relative to the electron spectrometer; Fig. 6(b) displays the calculated Fermi contours for comparison. The FS-as seen in the experiment-consists of a central ring created by band $3 \uparrow$ that is surrounded by a hexagon from band $3 \downarrow$; both features represent hole pockets. The hexagon has bright corners, which are located at the $\mathbf{k}$ positions, where band $2 \downarrow$ crosses

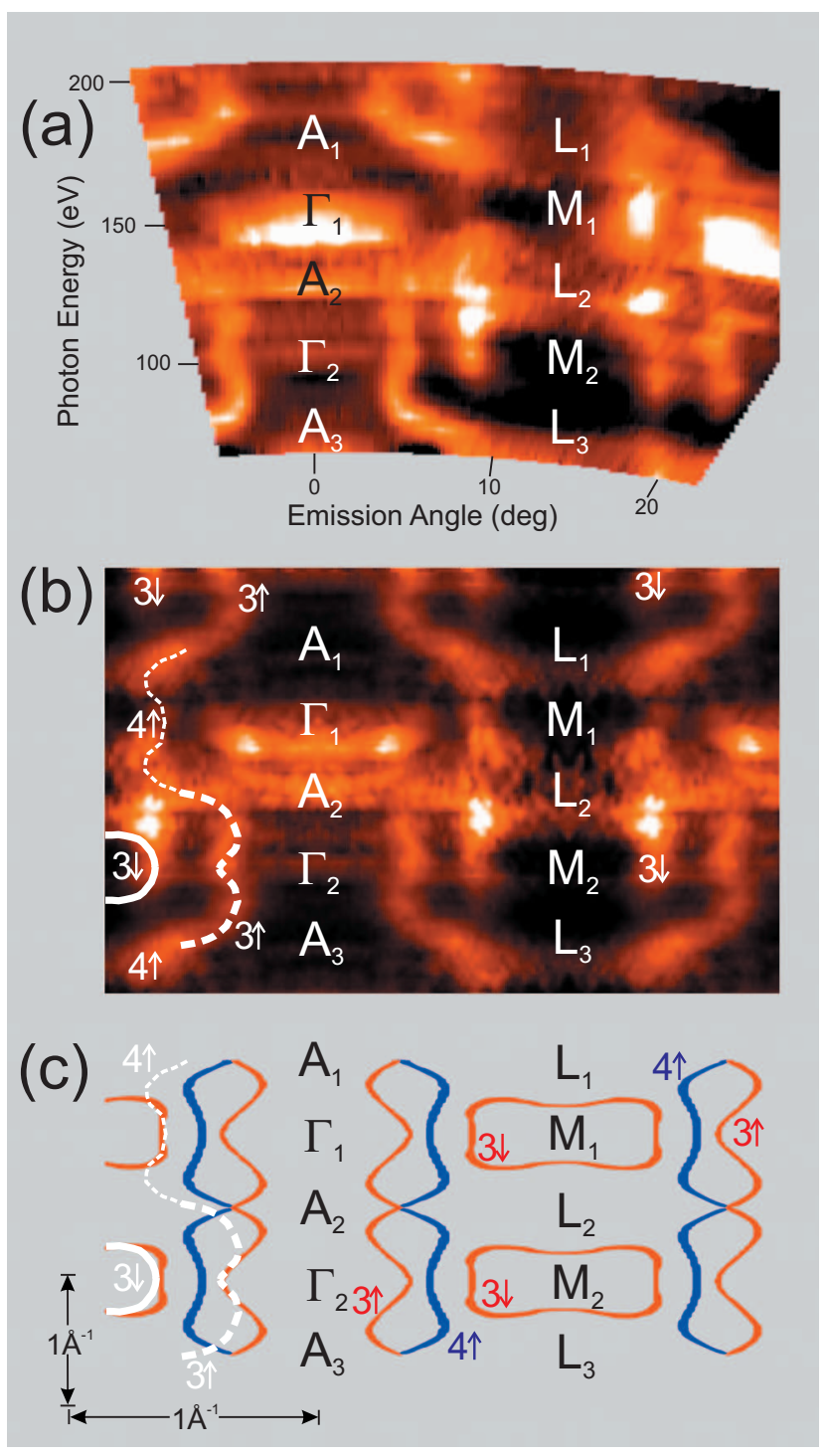

FIG. 7. (Color) Fermi contours in the $\Gamma A L M$ plane, i.e., perpendicular to the sample surface: (a) Data as measured as a function of photon energy (ordinate) and emission angle relative to the surface normal (abscissa); bright colors correspond to high PE intensities. In the present experiments, it is difficult to resolve Fermi contours in the region from $\Gamma_{1} M_{1}$ to $A_{2} L_{2}$, where the photon energy is passing through the $\mathrm{Tb} 4 d \rightarrow 4 f$ giant resonance, with a variation of the PE intensity by several orders of magnitude. To obtain the Fermi contours as shown in (b), autocorrelation operations combined with symmetries of the hcp lattice were applied on the as-measured data (for details see text). $\Delta_{2}$-like contours of bands $3 \uparrow$ and $3 \downarrow$ as well as the $\Delta_{1}$-like band $4 \uparrow$ are clearly observed. The white lines represent guides to the eyes and highlight the FS features. (c) Theoretical results for the Fermi surface of Tb metal, where the white lines on the left-hand side of the panel represent the experimental Fermi contours. The agreement along $\Gamma M$ for bands $3 \uparrow$ and $3 \downarrow$ is remarkably good, while some deviations show up toward $A L$. Note that in (b) and (c) the scales on abscissa and ordinate differ by a factor of 2 .

$E_{F}$ [see also Fig. 2(a), $\Gamma K$ section]. The experimental contours repeat themselves with less intensity in the adjacent $\mathrm{BZ}$, an observation that can be ascribed to matrix element 
effects. Features at $E_{F}$ caused by band $4 \uparrow\left(\Delta_{1}\right.$-like symmetry), which is marked in blue color in Fig. 6(b), are not observed at $h \nu \simeq 105 \mathrm{eV}$. Around $K$, the $\Delta_{2}$-like band $2 \downarrow$ and the $\Delta_{1}$-like band $1 \downarrow$ cross $E_{F}$, as indicated by green color in (b). The agreement between experiment and theory in the $\Gamma M K$ plane is remarkably good. A comparison of the present results with those of Ref. 21 reveals good agreement in the description of the Fermi contours due to bands $3 \uparrow$ and $4 \uparrow$, while for bands $2 \downarrow$ and $3 \downarrow$ quite differently shaped contours are obtained in the two approaches.

Figure 7 shows the Fermi surface measured along the ГALM azimuth; it highlights the nature of the dispersion along $k_{z}$. The experimental data in Fig. 7(a) were obtained from measurements performed over a much wider energy range $(80-200 \mathrm{eV})$ as compared to those presented in Fig. 6(a) for constant $k_{z}$. This implies a passing through the $\mathrm{Tb}$ $4 d \rightarrow 4 f$ giant resonance around $h \nu=150 \mathrm{eV}$, which gives rise to strong increases in the PE intensity and as a consequence to the bright areas in the as-measured data around $\Gamma_{1} A_{2}$ [see Fig. 7(a)]; Table III lists the corresponding photon energies for the $\Gamma$ and $A$ points.

To enhance the signal-to-noise ratio and to suppress effects of resonant PE, various autocorrelation operations were used, taking the symmetry of the BZ into account. The following symmetry operations of the hcp lattice were considered: (i) mirroring at the $\Gamma A$ and $\Gamma M$ lines; (ii) translation by the distance $\Gamma \Gamma$ along $\Gamma M$ and translation of 2 times the $\Gamma \Gamma$ distance along $\Gamma A$ (this accounts for the $\Delta_{1}$ and $\Delta_{2}$ selectivity of PE measurements). Specifically, if the measured PE intensity is $I_{1}$ at a certain point $\mathbf{k}_{1}$ of the $\mathrm{BZ}$, and $I_{2}$ at the corresponding identical point $\mathbf{k}_{2}$, it is replaced at both points by $I_{a c}=\left(I_{1} I_{2}\right)^{1 / 2}$. This autocorrelation treatment of the asmeasured data results in the Fermi contours shown in Fig. 7(b); for clarity, the autocorrelated PE intensities were copied-according to the symmetry of the BZ-to those areas that are not covered by the image shown in Fig. 7(a).

At the top of Fig. 7(b), above $A_{1} L_{1}$, Fermi contours of bands $3 \uparrow$ and $3 \downarrow$ are visible; band $3 \uparrow$ crosses over to band $4 \uparrow$ at $A_{1} L_{1}$ (no backfolding due to the $\Delta_{1}$ and $\Delta_{2}$ selectivity), and continues as band $4 \uparrow$ towards $\Gamma_{1} M_{1}$. The region above $\Gamma_{1} M_{1}$ in (b) was obtained from autocorrelation operations of the PE intensities measured in the photon-energy region from 80 to $105 \mathrm{eV}$ with those measured in the region from 160 to $200 \mathrm{eV}$ (here the translational symmetry of 2 times the $Г \Gamma$ distance along the $k_{z}$ direction was applied). Between $\Gamma_{1} M_{1}$ and $A_{2} L_{2}$ (with photon energies between 140 and $160 \mathrm{eV}$ ), strong intensity variations are observed, caused by the Tb $4 d \rightarrow 4 f$ giant resonance. Moreover, some Fermi-surface features of bands with $\Delta_{2}$-like symmetry are also observed, e.g., in the vicinity of $\Gamma_{1}$ [possible reasons have been addressed further above in the discussion of Fig. 2(b)]. Below $A_{2} L_{2}$ ( $\Delta_{2}$-like symmetry), $3 \uparrow$ and $3 \downarrow$ are visible, and below $\Gamma_{2} M_{2}$, the Fermi contours from the top of the image are repeated, due to the autocorrelation operation in the $k_{z}$ direction. The white lines in Fig. 7(b), which serve as guides to the eyes, indicate the evolution of the Fermi surface: The solid white line represents the $3 \downarrow$ band and the dashed white lines represent the $3 \uparrow$ (heavy) and the $4 \uparrow$ (light) bands. Figure 7(c) shows the corresponding theoretical Fermi contours, where the white lines mark the measured
TABLE III. Photon energies (in $\mathrm{eV}$ ) for $\Gamma$ and $A$ points shown in Fig. 7(a).

\begin{tabular}{lccccc}
\hline \hline Symmetry point & $A_{1}$ & $\Gamma_{1}$ & $A_{2}$ & $\Gamma_{2}$ & $A_{3}$ \\
\hline Photon energy & 183 & 155 & 129 & 106 & 85 \\
\hline \hline
\end{tabular}

Fermi contours from (b). Starting from the same position as the measured features along $\Gamma_{2} M_{2}$, the calculated $3 \uparrow$ band disperses too close to the $\Gamma A$ line toward $A_{2} L_{2}$. This tendency is passed on to the $4 \uparrow$ feature above $A_{2} L_{2}$. We summarize that the general shape of the Fermi surface of Tb metal on the $\Gamma A L M$ azimuth, shown in (b), is reproduced quite well by the calculations.

\section{CONCLUSIONS}

For ferromagnetic $\mathrm{Tb}$ metal, extensive PE data on the electronic band structure along several high-symmetry directions of the hexagonal BZ, as well as on Fermi contours, have been measured including critical points and Fermi vectors. In addition, state-of-the-art band-structure calculations were carried out, which achieve a remarkably good agreement with experiment, particularly for the $\Gamma M K$ plane. This represents a significant improvement compared to previous attempts to theoretically describe the electronic structure of ferromagnetic $\mathrm{Tb}$ metal. However, some systematic deviations between experiment and theory are still noticeable, e.g., an overestimation of the exchange splitting as well as a shift towards lower $\left(E-E_{F}\right)$ values at the critical points; these shifts are smaller for minority electronic states than for majority states. From the comparison presented in this work, we expect an improvement of the theoretical understanding of the electronic properties of $4 f$ metals and its possible inclusion in future theoretical models.

We have also shown that ARPES, as performed at beamline 7.0.1 of the ALS, is highly suitable for investigating the four-dimensional electronic structure of a solid over a wide range of momentum and binding-energy space, including several BZs. The relatively short time required for data taking — only a few hours for a full dataset—opens the possibility to perform temperature-dependent studies, which are expected to reveal changes of the FS across a magnetic phase transition of a given lanthanide metal. In this way, open questions concerning the interplay between the FS and the magnetic ordering in these materials, including the magnetic structure, can be addressed.

\section{ACKNOWLEDGMENTS}

This work was supported by the Bundesministerium für Bildung und Forschung, Federal Republic of Germany, Contract No. $05 \mathrm{KS} 1 \mathrm{KEC} / 2$, the Deutsche Forschungsgemeinschaft, Contracts No. STA 413/3-1 and No. BI 823/1-1, and the U.S. Department of Energy under Contract No. DEAC03-76SF00098. One of the authors (J.E.P.) gratefully acknowledges the support by the programme Ramón y Cajal of the Spanish MEC. 
*doebrich@physik.fu-berlin.de

tDeceased.

Fresent address: Centro de Microanalisis de Materiales, Universidad Autonoma de Madrid, 28049 Madrid, Spain.

${ }^{\S}$ Present address: Institut für Experimentelle und Angewandte Physik, Universität Kiel, 24098 Kiel, Germany.

${ }^{1}$ A. J. Freeman, in Magnetic Properties of Rare Earth Metals, edited by R. J. Elliott (Plenum, London, 1972), pp. 245-333.

${ }^{2}$ S. Legvold, in Magnetic Properties of Rare Earth Metals, edited by R. J. Elliott (Plenum, London, 1972), pp. 335-381.

${ }^{3} \mathrm{~S}$. H. Liu, in Handbook of the Physics and Chemistry of Rare Earths, edited by K. A. Gschneidner, Jr. and L. R. Eyring (North-Holland, Amsterdam, 1978), Vol. 1, pp. 233-335.

${ }^{4}$ J. Jensen and A. R. Mackintosh, Rare Earth Magnetism (Clarendon, Oxford, 1991).

${ }^{5}$ F. J. Himpsel and B. Reihl, Phys. Rev. B 28, 574 (1983).

${ }^{6}$ C. Waldfried, T. McAvoy, D. Welipitiya, T. Komesu, P. A. Dowben, and E. Vescovo, Phys. Rev. B 58, 7434 (1998).

${ }^{7}$ B. Kim, A. B. Andrews, J. L. Erskine, K. J. Kim, and B. N. Harmon, Phys. Rev. Lett. 68, 1931 (1992).

${ }^{8}$ K. Maiti, M. C. Malagoli, E. Magnano, A. Dallmeyer, and C. Carbone, Phys. Rev. Lett. 86, 2846 (2001).

${ }^{9}$ K. Maiti, M. C. Malagoli, A. Dallmeyer, and C. Carbone, Phys. Rev. Lett. 88, 167205 (2002).

${ }^{10} \mathrm{Ph}$. Kurz, G. Bihlmayer, and S. Blügel, J. Phys.: Condens. Matter 14, 6353 (2002).

${ }^{11}$ W. Nolting, Z. Phys. B: Condens. Matter 94, 409 (1994).

${ }^{12}$ C. Santos, W. Nolting, and V. Eyert, Phys. Rev. B 69, 214412 (2004).

${ }^{13}$ L. M. Sandratskii and J. Kübler, in Magnetism and Electronic Correlations in Local-Moment Systems: Rare-Earth Elements and Compounds, edited by M. Donath, P. A. Dowben, and W. Nolting (World Scientific, Singapore, 1998), pp. 271-291.

${ }^{14}$ C. Schüßler-Langeheine, E. Weschke, C. Mazumdar, R. Meier, A. Y. Grigoriev, G. Kaindl, C. Sutter, D. Abernathy, G. Grübel, and M. Richter, Phys. Rev. Lett. 84, 5624 (2000).

${ }^{15}$ C. Schüßler-Langeheine, E. Weschke, H. Ott, A. Y. Grigoriev, A. Möller, R. Meier, C. Mazumdar, and G. Kaindl, J. Electron Spectrosc. Relat. Phenom. 114, 795 (2001).

${ }^{16}$ E. Weschke and G. Kaindl, J. Phys.: Condens. Matter 13, 11133 (2001).

${ }^{17}$ R. I. R. Blyth, S. D. Barrett, S. S. Dhesi, R. Cosso, N. Heritage, A. M. Begley, and R. G. Jordan, Phys. Rev. B 44, 5423 (1991).

${ }^{18}$ S. C. Wu, H. Li, D. Tian, J. Quinn, Y. S. Li, F. Jona, J. Sokolov, and N. E. Christensen, Phys. Rev. B 41, 11911 (1990) [see also Phys. Rev. B 43, 12060 (1991)].

${ }^{19}$ S. C. Wu, H. Li, Y. S. Li, D. Tian, J. Quinn, F. Jona, D. Fort, and N. E. Christensen, Phys. Rev. B 45, 8867 (1992).

${ }^{20}$ C. Jackson, Phys. Rev. 178, 949 (1969).

${ }^{21}$ R. Ahuja, S. Auluck, B. Johansson, and M. S. S. Brooks, Phys. Rev. B 50, 5147 (1994).

${ }^{22}$ L. Nordström and A. Mavromaras, Europhys. Lett. 49, 775 (2000).

${ }^{23}$ S. D. Barrett and R. G. Jordan, Z. Phys. B: Condens. Matter 66, 375 (1987).

${ }^{24}$ S. D. Barrett, R. G. Jordan, A. M. Begley, and R. I. R. Blyth, Z. Phys. B: Condens. Matter 76, 137 (1989).

${ }^{25}$ S. B. Dugdale, H. M. Fretwell, M. A. Alam, G. Kontrym-Sznajd, R. N. West, and S. Badrzadeh, Phys. Rev. Lett. 79, 941 (1997).

${ }^{26}$ H. M. Fretwell, S. B. Dugdale, M. A. Alam, D. C. R. Hedley, A.
Rodriguez-Gonzalez, and S. B. Palmer, Phys. Rev. Lett. 82, 3867 (1999).

${ }^{27}$ S. J. Crowe, S. B. Dugdale, Z. Major, M. A. Alam, J. A. Duffy, and S. B. Palmer, Europhys. Lett. 65, 235 (2004).

${ }^{28}$ L. Petit, A. Svane, Z. Szotek, and W. M. Temmerman, Phys. Rev. B 72, 205118 (2005).

${ }^{29}$ V. I. Anisimov, F. Aryasetiawan, and A. I. Lichtenstein, J. Phys.: Condens. Matter 9, 767 (1997).

${ }^{30}$ P. Larson, W. R. L. Lambrecht, A. Chantis, and M. van Schilfgaarde, Phys. Rev. B 75, 045114 (2007).

${ }^{31}$ U. Lundin, I. Sandalov, and O. Eriksson, Int. J. Quantum Chem. 102, 1046 (2005)

${ }^{32}$ A. V. Andrianov, O. A. Savel'eva, E. Bauer, and C. Paul, Phys. Rev. B 72, 132408 (2005).

${ }^{33}$ F. Heigl, J. E. Prieto, O. Krupin, K. Starke, G. Kaindl, and M. Bode, Phys. Rev. B 72, 035417 (2005).

${ }^{34}$ J. P. Perdew, K. Burke, and M. Ernzerhof, Phys. Rev. Lett. 77, 3865 (1996).

${ }^{35}$ E. Wimmer, H. Krakauer, M. Weinert, and A. J. Freeman, Phys. Rev. B 24, 864 (1981).

${ }^{36}$ M. Weinert, E. Wimmer, and A. J. Freeman, Phys. Rev. B 26, 4571 (1982).

${ }^{37}$ C. Li, A. J. Freeman, H. J. F. Jansen, and C. L. Fu, Phys. Rev. B 42, 5433 (1990).

${ }^{38}$ D. Singh, Phys. Rev. B 43, 6388 (1991).

${ }^{39}$ M. H. Cohen and L. M. Falicov, Phys. Rev. Lett. 5, 544 (1960).

${ }^{40}$ F. J. Himpsel and D. E. Eastman, Phys. Rev. B 21, 3207 (1980) [see also Phys. Rev. B 22, 5014 (1980)].

${ }^{41}$ D. Pescia, A. R. Law, M. T. Johnson, and H. P. Hughes, Solid State Commun. 56, 809 (1985).

${ }^{42}$ R. L. Benbow, Phys. Rev. B 22, 3775 (1980).

${ }^{43}$ W. Lenth, F. Lutz, J. Barth, G. Kalkoffen, and C. Kunz, Phys. Rev. Lett. 41, 1185 (1978).

${ }^{44}$ K. Starke, Z. Hu, F. Hübinger, E. Navas, G. Kaindl, and G. van der Laan, Eur. Phys. J. B 12, 171 (1999).

${ }^{45}$ A. V. Fedorov, T. Valla, F. Liu, P. D. Johnson, M. Weinert, and P. B. Allen, Phys. Rev. B 65, 212409 (2002).

${ }^{46}$ D. Wegner, A. Bauer, and G. Kaindl, Phys. Rev. B 73, 165415 (2006).

${ }^{47}$ E. Weschke, C. Schüssler-Langeheine, R. Meier, A. V. Fedorov, K. Starke, F. Hübinger, and G. Kaindl, Phys. Rev. Lett. 77, 3415 (1996).

${ }^{48}$ D. E. Hegland, S. Legvold, and F. H. Spedding, Phys. Rev. 131, 158 (1963).

${ }^{49}$ N. V. Smith, Comments Condens. Matter Phys. 15, 263 (1992).

${ }^{50}$ J. A. Knapp, F. J. Himpsel, and D. E. Eastman, Phys. Rev. B 19, 4952 (1979).

${ }^{51}$ M. P. Seah and W. A. Dench, Surf. Interface Anal. 1, 2 (1979).

${ }^{52}$ N. V. Smith, P. Thiry, and Y. Petroff, Phys. Rev. B 47, 15476 (1993).

${ }^{53}$ J. C. Slater, Quantum Theory of Molecules and Solids (McGrawHill, New York, 1965), Vol. 2.

${ }^{54}$ S. V. Borisenko et al., Phys. Rev. B 64, 094513 (2001).

${ }^{55}$ L. Kipp, K. Roßnagel, C. Solterbeck, T. Strasser, J. W. Schattke, and M. Skibowski, Phys. Rev. Lett. 83, 5551 (1999).

${ }^{56}$ N. W. Ashcroft and N. D. Mermin, Solid State Physics (Saunders College, Philadelphia, 1976).

${ }^{57}$ S. D. Kevan, Phys. Scr., T T31, 32 (1990).

${ }^{58}$ A. Santoni, L. J. Terminello, F. J. Himpsel, and T. Takahashi, Appl. Phys. A: Solids Surf. 52, 299 (1991). 